\title{
The use of Lecture Capture in
} Light of Teaching Approach and Content Type: An Institution-Wide Study

Jared Danielson, Holly Bender, Lesya Hassall, Vanessa Preast

\author{
Mar 10, 2012 \\ AAVMC
}




\section{Lecture Capture (in our context)}

- Each full course session captured

- Audio of instructor

- Video of whatever is displayed on the computer

- Video (small) of the instructor

- Podcast and Vodcast available (instructor preference) 


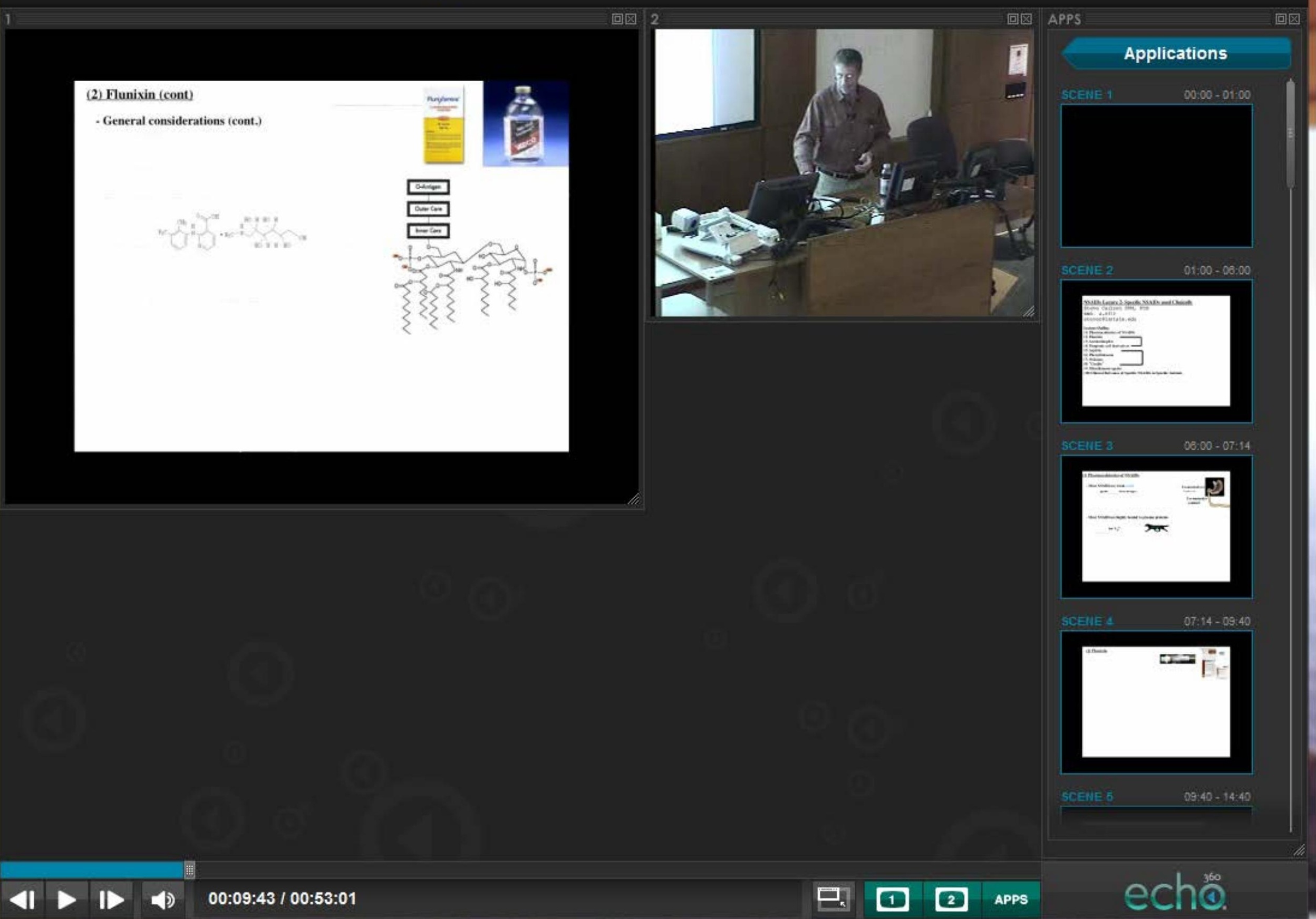




\section{(4) Propionic acid derivatives (profens)}

\begin{abstract}
(A) Ibuprofen
(B) Ketoprofen

(C) Carprofen

(D) Naproxen
\end{abstract}

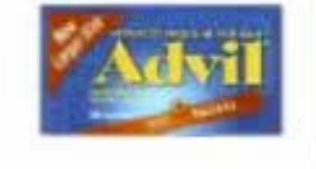

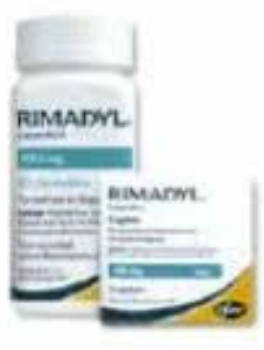

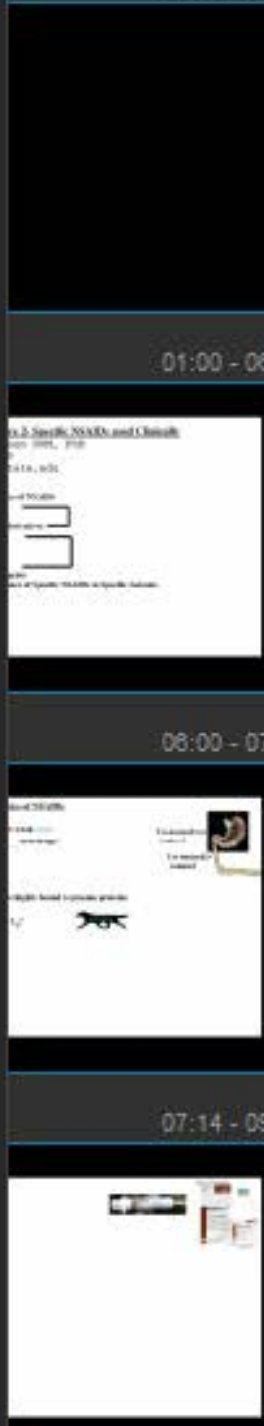

- non-selective: except for carprofen

(A) Ibuprofen: not safe in veterinary patients

(B) Ketoprofen:

- COX inhibitor and a partial LOX inhibitor

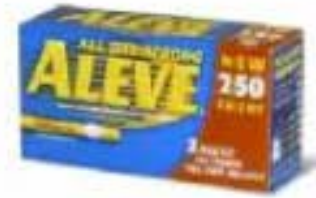




\section{Echo 360 Use at the ISUCVM}

- Students self-reported access, on average (per survey):

$\begin{array}{ccc}\begin{array}{c}\text { Average } \\ \text { Week }\end{array} & \begin{array}{c}\text { Heavy Use } \\ \text { Week }\end{array} & \begin{array}{c}\text { Light Use } \\ \text { Week }\end{array}\end{array}$

Average

2.4

5.3

1.1

$\operatorname{Max}$

10

30

6

Min

0

0

0 


\section{Why do students use captured lectures?}

- Review key points

- Obtain additional clarity

- Make up for unavoidable conflicts

- Review for tests

(Simpson, 2006; Wilson \& Weiser, 2001; Winer \& Cooperstock, 2002; Yudko, Hirokawa, \& Chi, 2008)

- Provide quality audio and ability to pause/replay for $2^{\text {nd }}$ language learners

(Simpson, 2006) 


\section{Research Questions}

What is the relationship between...

1. instructor teaching approach and the attitudes that instructors and students have towards lecture capture?

2. course content type and the attitudes that instructors and students have towards lecture capture?

3. use of lecture capture and learning outcomes? Is this relationship associated with teaching approach or course content? 


\section{Population}

- Survey sent to 565 veterinary students

(yrs. 1-4, 75\% female; 25\% male)

- Respondents: 222 (39\%)

Self-reported use of Echo:

- Have you ever reviewed lectures captured by

Echo?

- $218(98.2 \%)-Y e s$

- $4(1.8 \%)-$ No 


\section{Teaching Approach Hypotheses and Results}

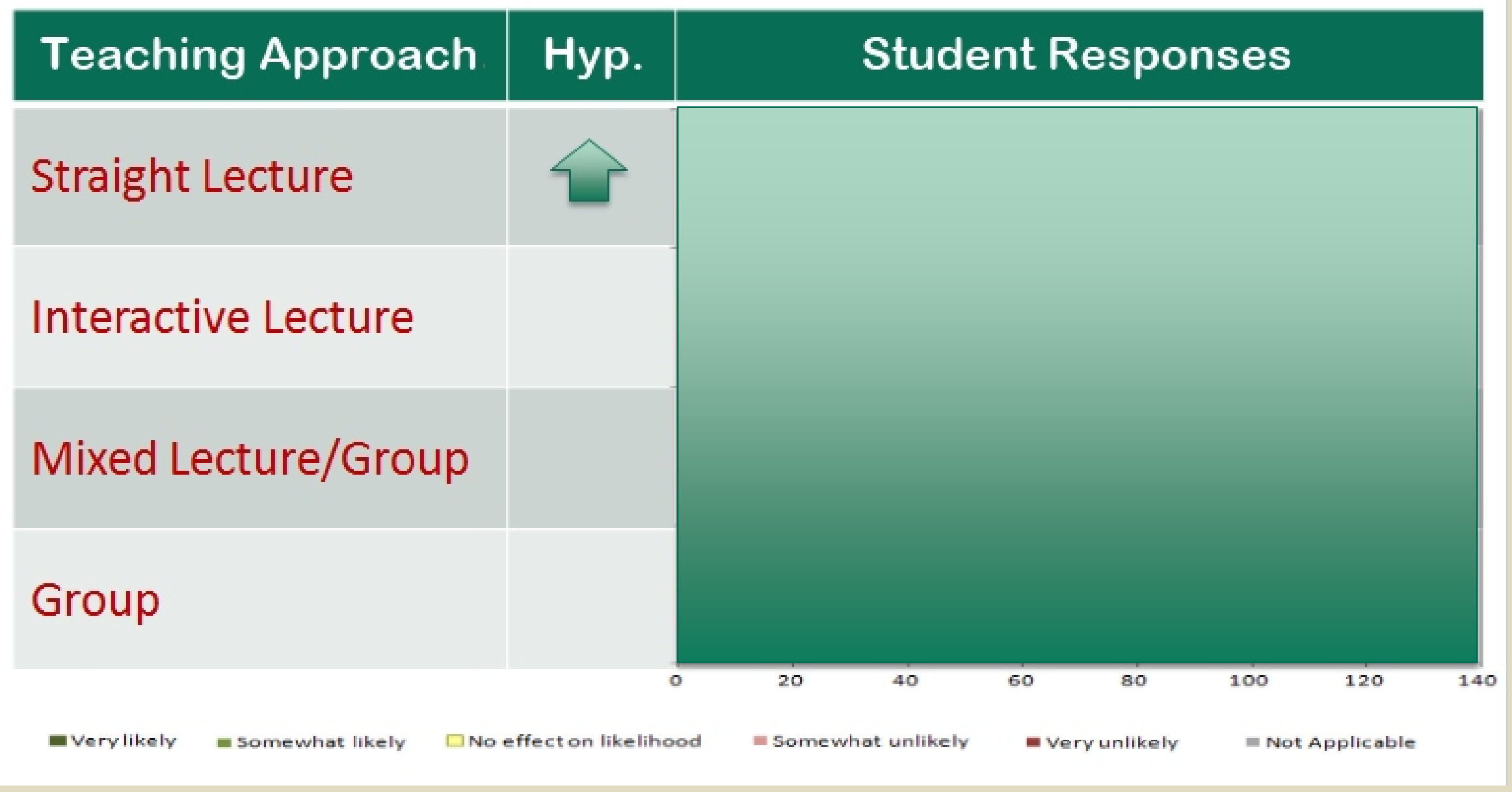

The teaching approaches were significantly different from each other [Wilcoxon Test. $-(p<0.0005)$ ]

Friedman test for non-parametric data $(\mathrm{N}=191$, Chi-Square 429.567, df = 3, Sig < 0.0005); 


\section{Teaching Approach Hypotheses and Results}

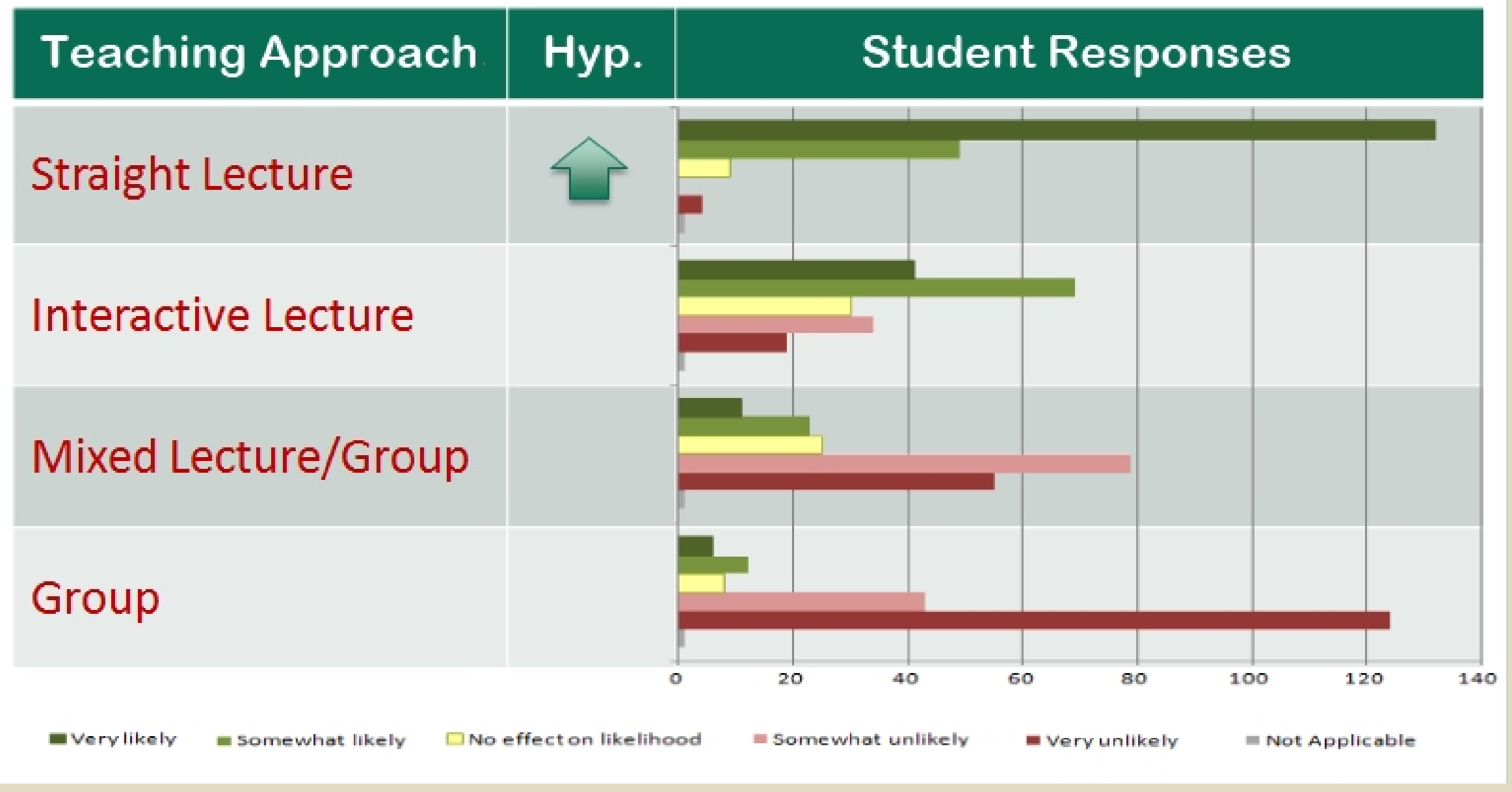

The teaching approaches were significantly different from each other [Wilcoxon Test. $-(p<0.0005)$ ]

Friedman test for non-parametric data $(\mathrm{N}=191$, Chi-Square 429.567, df = 3, Sig < 0.0005); 


\section{Curricular Coordination Hypotheses and Results}

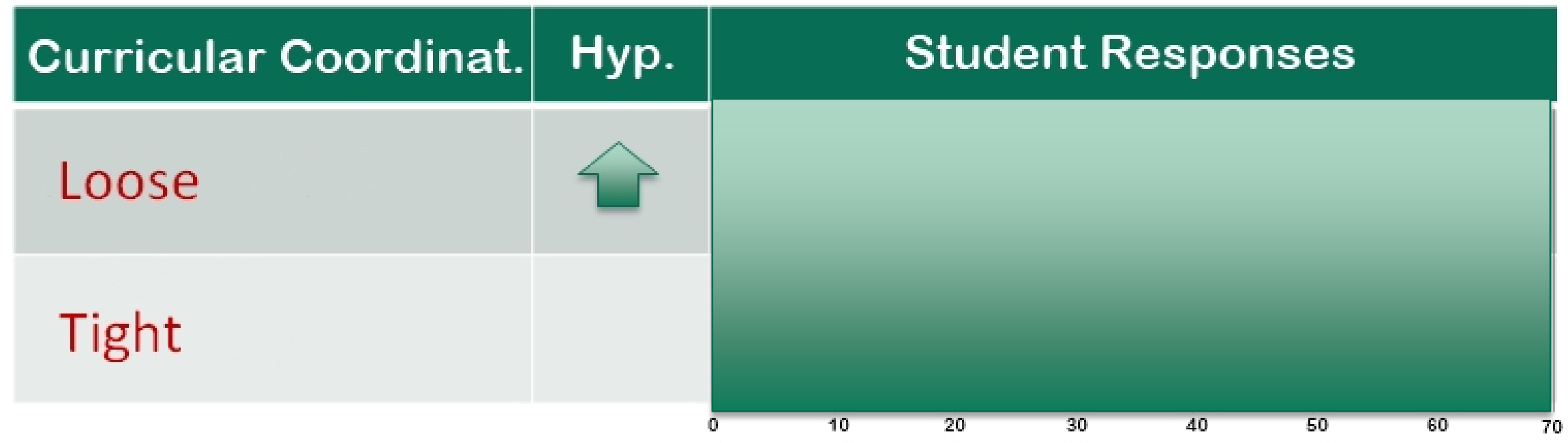

Very likely $\quad$ Somewhat likely $\square$ No effect on likelihood $\quad \square$ Somewhat unlikely $\quad$ Very unlikely $\quad$ NA 


\section{Curricular Coordination Hypotheses and Results}

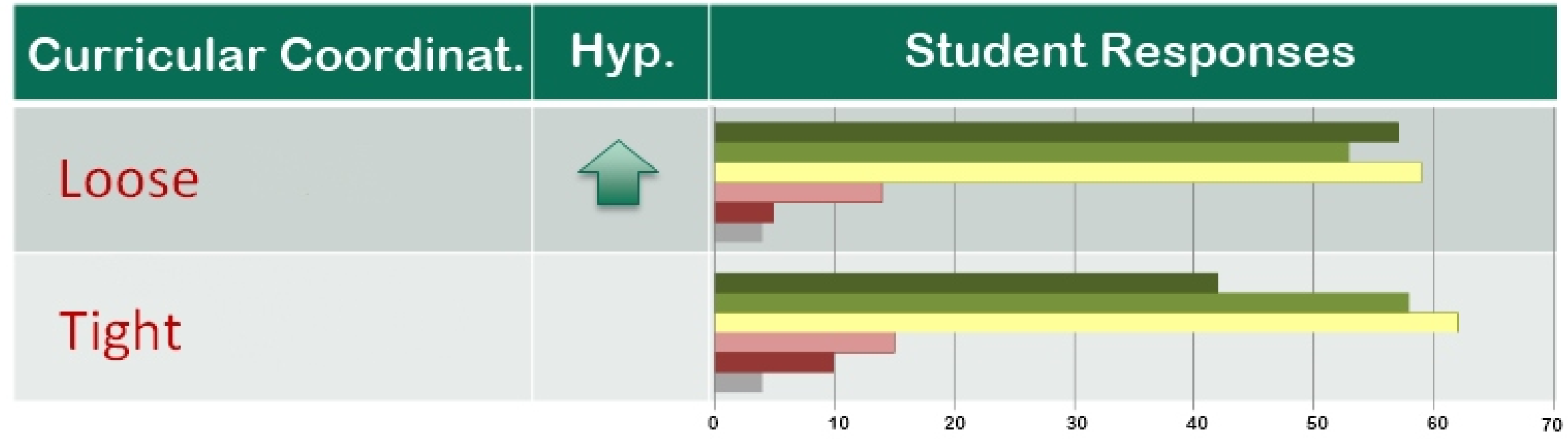

Verylikely $\quad$ Somewhat likely $\square$ No effect on likelihood $\square$ Somewhat unlikely $\quad$ Very unlikely $\quad$ NA 


\section{Content \\ Hypotheses and Results}

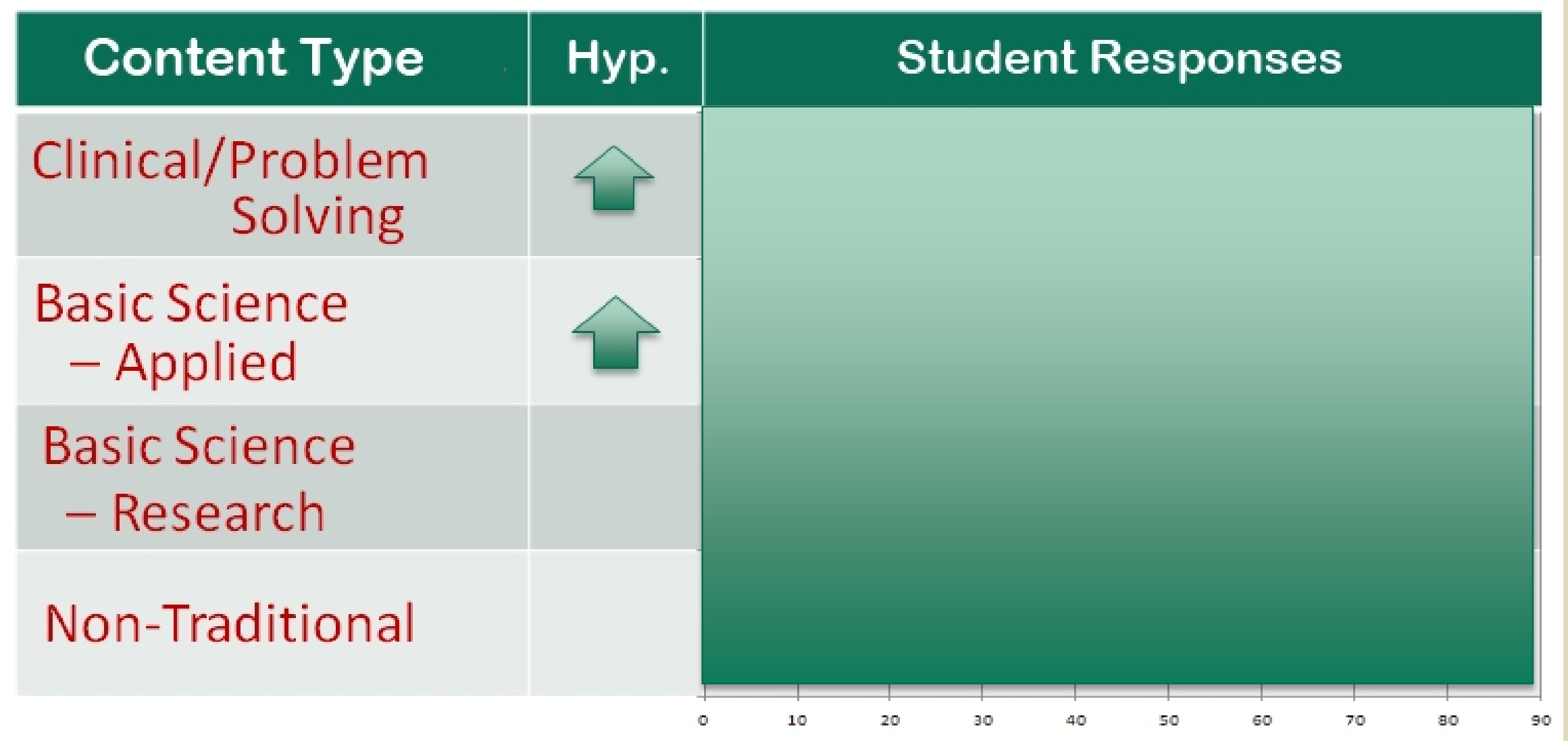

Verylikely $\quad$ Somewhat likely $\square$ No effect on likelihood $\square$ Somewhat unlikely $\quad$ Very unlikely $\quad$ NA

The Non-Traditional classes were significantly different from all others [Wilcoxon Signed Ranks Test ].

Friedman Test $(N=181$, Chi-Square $=229.810, \mathrm{df}=3, \mathrm{p}<.0005)$; 


\section{Content \\ Hypotheses and Results}

\section{Content Type}

Hyp.

Clinical/Problem Solving

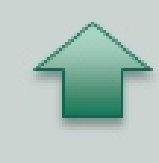

Basic Science

- Applied

Basic Science

- Research

Non-Traditional

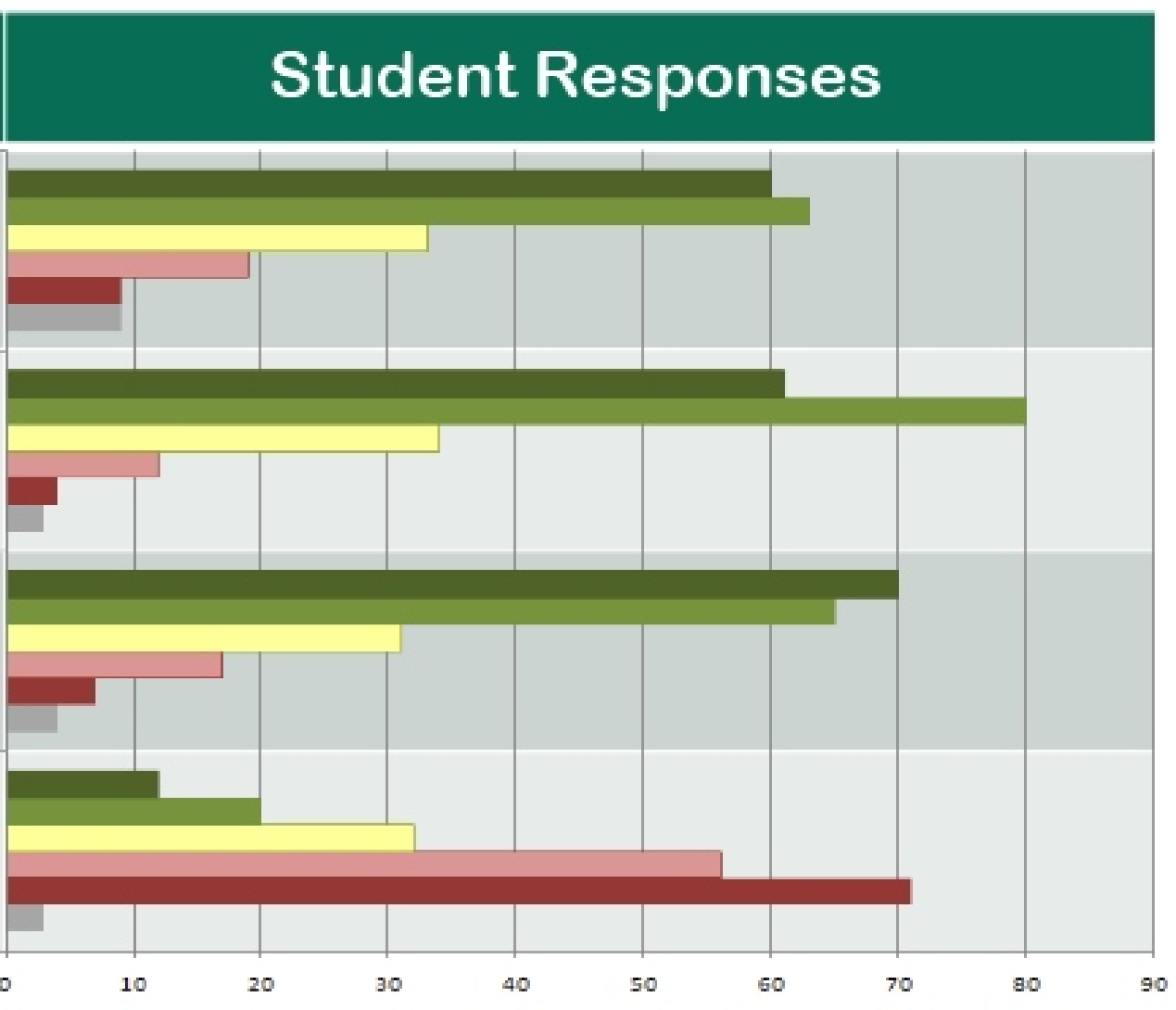

घerylikely $\square$ Somewhat likely $\square$ No effect on likelihood $\square$ Somewhat unlikely $\quad$ Very unlikely $\square$ NA

The Non-Traditional classes were significantly different from all others [Wilcoxon Signed Ranks Test ].

Friedman Test $(\mathrm{N}=181$, Chi-Square $=229.810, \mathrm{df}=3, \mathrm{p}<.0005)$; 


\section{Frequent* Important Purposes: \\ $(n=199-201)$}

\section{Purpose/Condition}

\section{Average}

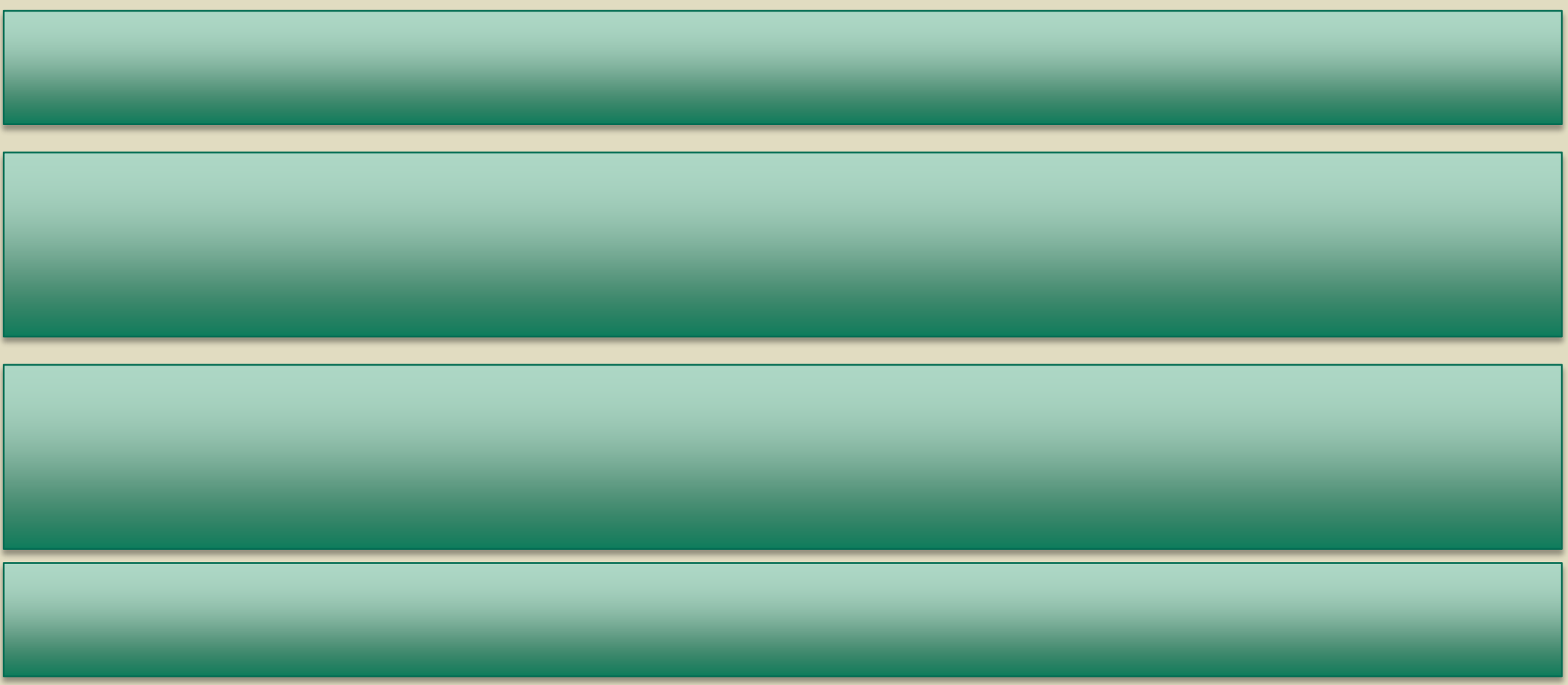

* One-Samples T-test, $p<.05$

Scale: 5 = Almost Always, $4=$ Freqently, $3=$ Neither Frequently nor Infrequently, $2=$ Infrequently, $1=$ almost never 


\section{Instructor/lecture characteristics that increase likelihood of accessing lectures}

\section{Characteristic}

Average

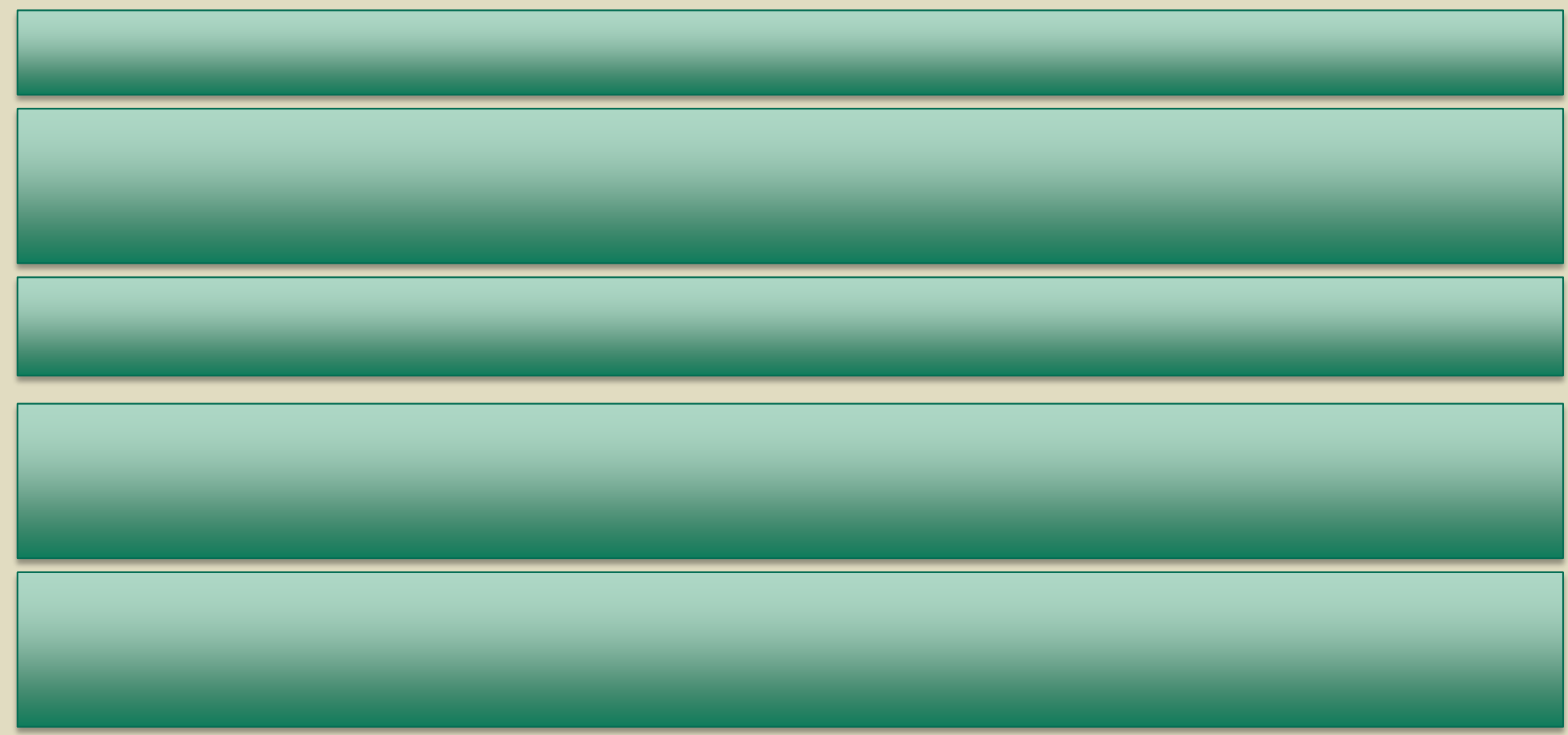

One sample t-test $\mathrm{P}<.05$

5 = Very Likely, 4 = Somewhat Likely, 3 = No effect on Likelihood, 2 = Somewhat Unlikely, 1 = Very Unlikely 


\section{Instructor/lecture characteristics that decrease likelihood of accessing lectures}

Characteristic

Average

One sample t-test $\mathrm{P}<.05$

5 = Very Likely, 4 = Somewhat Likely, 3 = No effect on Likelihood, $2=$ Somewhat Unlikely, 1 = Very Unlikely 
Instructor/lecture characteristics that do not increase or decrease students' self-reported likelihood of accessing lectures

- Skill as a lecturer

- Organization 


\section{Big Picture}

Relevance, Relevance, Relevance

- Strategic Use of Captures

- Appropriate visuals

- Relevant Content

- Missed Materials (not in notes, etc.)

- Test

Will this be helpful for review? 


\section{Faculty Survey}

- Sent to all instructional faculty

- 35 (48\%) responded

- Each faculty member responded for each class in which she/he taught 


\section{Instructor perspective - research questions}

- Faculty attitude towards lecture capture was not associated with teaching approach, curricular coordination, or content type. 


\section{Faculty Survey}

Question 1: What advantages does lecture capture provide you as an

$$
\text { instructor? }
$$

No Advantages perceived

Students can review the lectures

I (faculty) can review my own performance 


\section{Faculty Survey}

Question 4: What are the drawbacks to lecture capture for you as an

$$
\text { instructor? }
$$

$$
\text { and }
$$

Question 6: What do you like least about the lecture capture program at

\section{ISU?}

Lower class attendance

Students are less attentive or less likely to ask questions 


\section{Additional insight on faculty concerns ...}

- "The classroom dynamics are altered when student numbers drop below a certain point. The behavior of the INSTRUCTOR changes (based on personal experience and comments from other faculty members) when students choose to not be in the classroom. The absence of students probably has a significant effect on faculty performance... which hopefully is important in providing quality instruction."

- "... it [lecture capture] dramatically changes the classroom dynamics if a significant (?) percentage of students are absent from discussions/question \& answer sessions, etc." 


\section{Learning Outcomes}




\section{Prior Research}

- Studies have tended to focus on the difference in scores between those who utilized captured lectures for learning and those who didn't.

- Generally no significant differences were seen; where present, trends were inconsistent.

- Bacro, T. R. H., Gebregziabher, M., \& Fitzharris, T. P. (2010). Evaluation of a lecture recording system in a medical curriculum. Anatomical Sciences Education, 3, 300-308.

- Franklin, D. S., Gibson, J. W., Samuel, J. C., Teeter, W. A., \& Clarkson, C. W. (2011). Use of Lecture Recordings in Medical Education. Medical Science Educator, 1, 21-28. 


\section{Our question ...}

- Faculty (some) hypothesize - all ships sink together (i.e. you cannot just compare those who use captured lectures with those who don't). Does this happen?

- Do learning outcomes have to do with the "type" of course? 


\section{Instructor/Student perspective}

In general, are you (students) more likely to learn better with lecture capture technology?

\begin{tabular}{|c|c|c|c|c|}
\hline & \multicolumn{2}{|c|}{$\underline{\text { Students }}$} & \multicolumn{2}{|c|}{$\underline{\text { Faculty }}$} \\
\hline & $\underline{\mathrm{n}}$ & $\underline{\%}$ & $\underline{\mathrm{n}}$ & $\underline{\%}$ \\
\hline Very unlikely & 0 & $0.0 \%$ & 3 & $9.1 \%$ \\
\hline Somewhat unlikely & 1 & $0.5 \%$ & 3 & $9.1 \%$ \\
\hline No effect on likelihood & 11 & $5.4 \%$ & 15 & $45.5 \%$ \\
\hline Somewhat likely & 48 & $23.4 \%$ & 9 & $27.3 \%$ \\
\hline Very likely & 143 & $69.8 \%$ & 3 & $9.1 \%$ \\
\hline
\end{tabular}




\section{Participants}

- Veterinary students (classes of 2008 - 2112; n

= 614)

- $75 \%$ female; $25 \%$ male

- Average age - mid 20's. (All had completed 5 semesters of instruction at the time performance data were collected)

- All recruited using the same admissions criteria and having similar academic characteristics 


\section{Analysis of Outcomes Data}

- Dependent Variable

- Qualifying Examination (QE)

- Comprehensive Standardized Test of Basic Science Knowledge and Skills

- Offered after 5 semesters of instruction

-5 subscales

- Anatomy

- Physiology

- Pharmacology

- Pathology

- Microbiology 


\section{Descriptive lecture capture}

\section{"use" groupings}

- Independent Variable -- Based on the amount

of lecture captures viewed within the

discipline area across all courses in that

discipline over 5 semesters - (Views per

student per week)

- none (0 lectures accessed per week),

- low (.01- 1.3 views per week),

- medium (1.4 - 2.9 views per week) views per week,"

- high (> 2.9 views per week, on average.) 


\section{Class}

- Class used as a covariate to control for other curricular changes/fluctuations. 


\section{Views}

Class Anatomy Microbiology Pathology Pharmacology Physiology Overall

\begin{tabular}{|c|c|c|c|c|c|c|}
\hline 2008 & -- & -- & - & -- & -- & - \\
\hline 2009 & -- & -- & -- & -- & -- & -- \\
\hline 2010 & -- & -- & -- & 0.4 (Low) & 0.10 (Low) & 0.5 (Low) \\
\hline 2011 & -- & 2.6 (Med) & 0.5 (Low) & 1.9 (Med) & 0.3 (Low) & 5.2 (High) \\
\hline 2012 & 0.47 (Low) & 3.1 (High) & 0.6 (Low) & 1.6 (Med) & 0.3 (Low) & 6.1 (High) \\
\hline
\end{tabular}

Note: -- Means that no lectures were viewed, either because they were not available or the instructor did not allow viewing. 


\section{Results}

Class Anatomy Microbiology Pathology Pharmacology Physiology Overall

\begin{tabular}{|c|c|c|c|}
\hline 2008 & $\begin{array}{l}\text { No significant differences by lecture } \\
\text { views level }(F=.002 ; p=.998) \text { : }\end{array}$ & -- & -- \\
\hline 2009 & $\begin{array}{l}\text { None: } 226.9(44.5) \\
\text { Low: } 230.1(44.6)\end{array}$ & -- & -- \\
\hline 2010 & High: 233.9 (44.2) & 0.1 (Low) & 0.5 (Low) \\
\hline 2011 & $\begin{array}{l}\text { Covariate (class) not significant }(F=.398 ; \\
p=.531)\end{array}$ & 0.3 (Low) & 5.2 (High) \\
\hline 2012 & 1.6 (Med) & 0.3 (Low) & 6.1 (High) \\
\hline
\end{tabular}

Note: -- Means that no lectures were viewed, either because they were not available or the instructor did not allow viewing. 


\section{Low-Views, Basic Science Applied Disciplines}




\section{Physiology}

Class Anatomy Microbiology Pathology Pharmacology Physiology Overall

No significant differences by lecture views level $(F=.820 ; p=.366)$ :

None: $58.5(11.1)$

Low: 58.5 (10.1) $1.088 ; p=.297)$

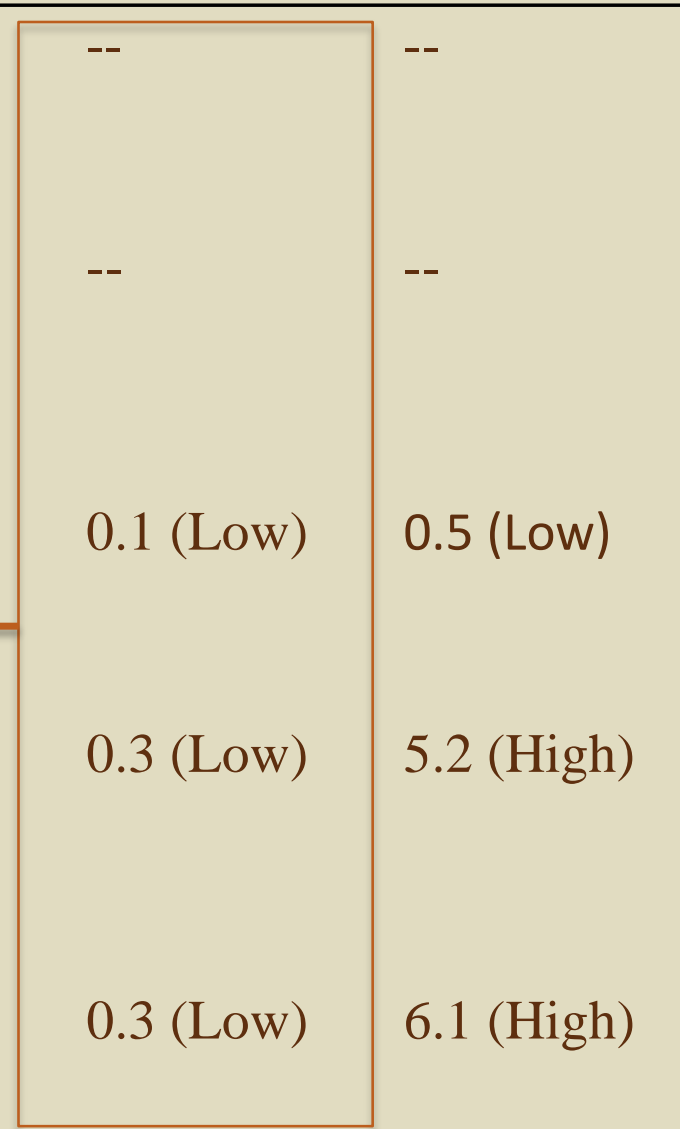

Note: -- Means that no lectures were viewed, either because they were not available or the instructor did not allow viewing. 


\section{Pathology}

Class Anatomy Microbiology Pathology Pharmacology Physiology Overall

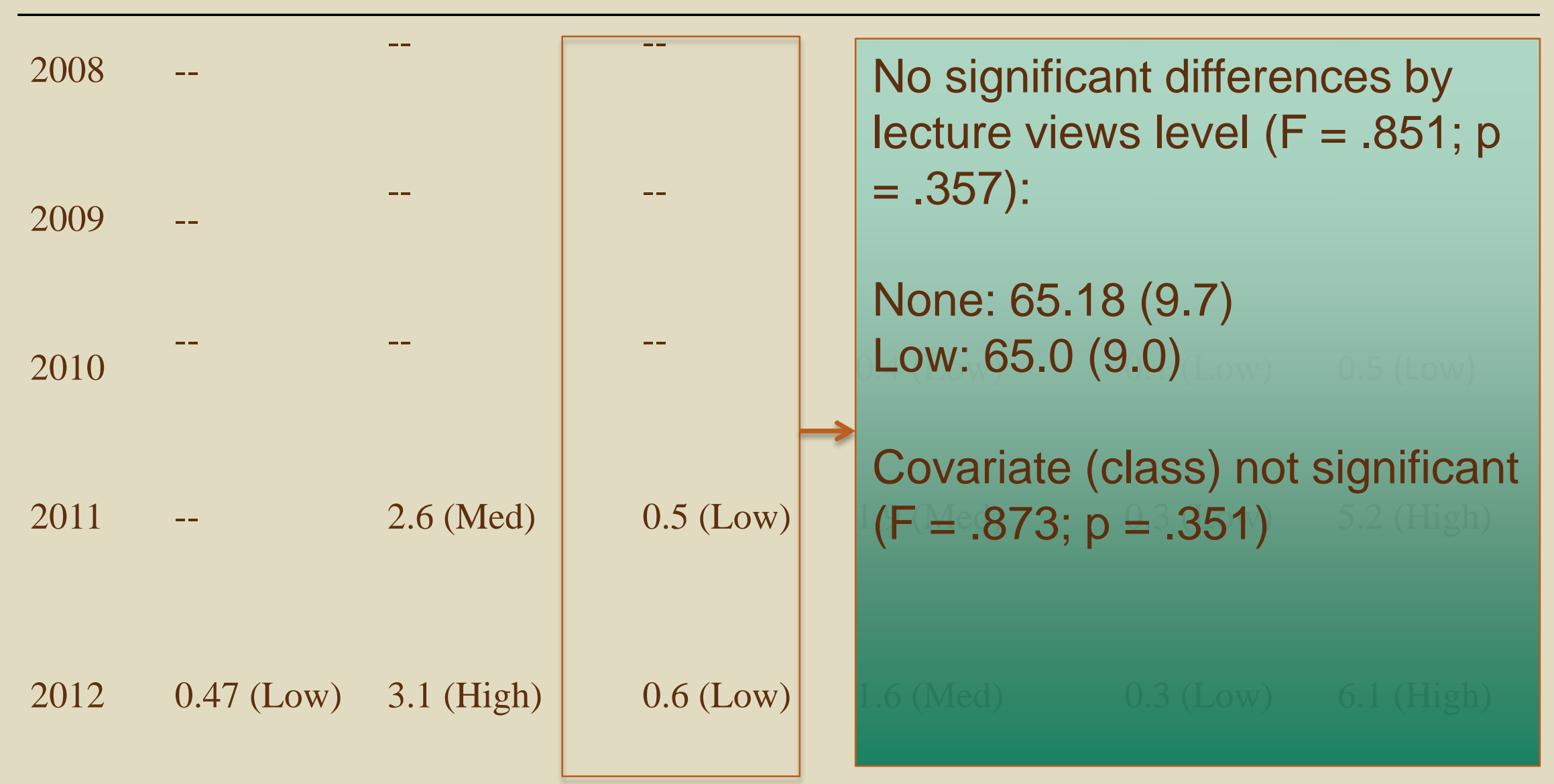

Note: -- Means that no lectures were viewed, either because they were not available or the instructor did not allow viewing. 
Higher/Student - requested views - Basic Science Research/Building block orientation 


\section{Pharmacology}

Class Anatomy Microbiology Pathology Pharmacology Physiology Overall

There were significant differences by lecture views level $(F=16.350$; $\mathrm{p}<.0005)$.

None: $51.7(11.3)$

Low: 52.5 (13.0)

Med: 59.5 (12.0)

Covariate (class) was also significant $(F=8.995 ; p=.003)$

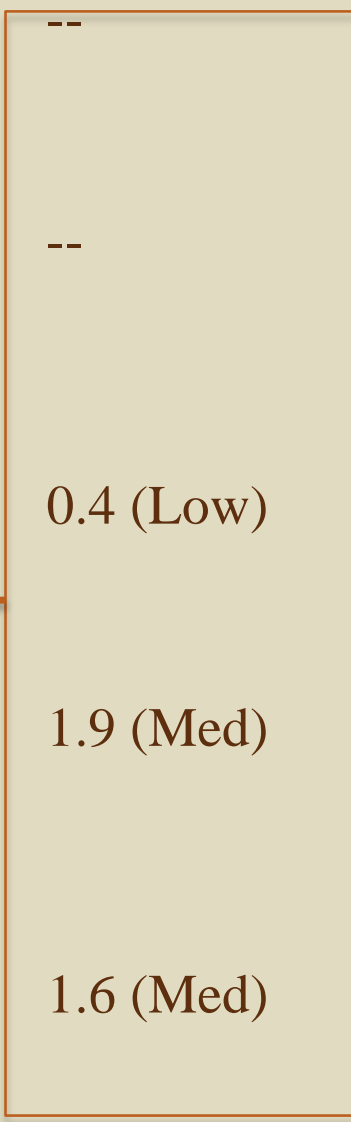

0.1 (Low) $\quad 0.5$ (Low)

0.3 (Low) 5.2 (High)

0.3 (Low) $\quad 6.1$ (High)

Note: -- Means that no lectures were viewed, either because they were not available or the instructor did not allow viewing. 


\section{Pharmacology}

Class Anatomy Microbiology Pathology Pharmacology Physiology Overall

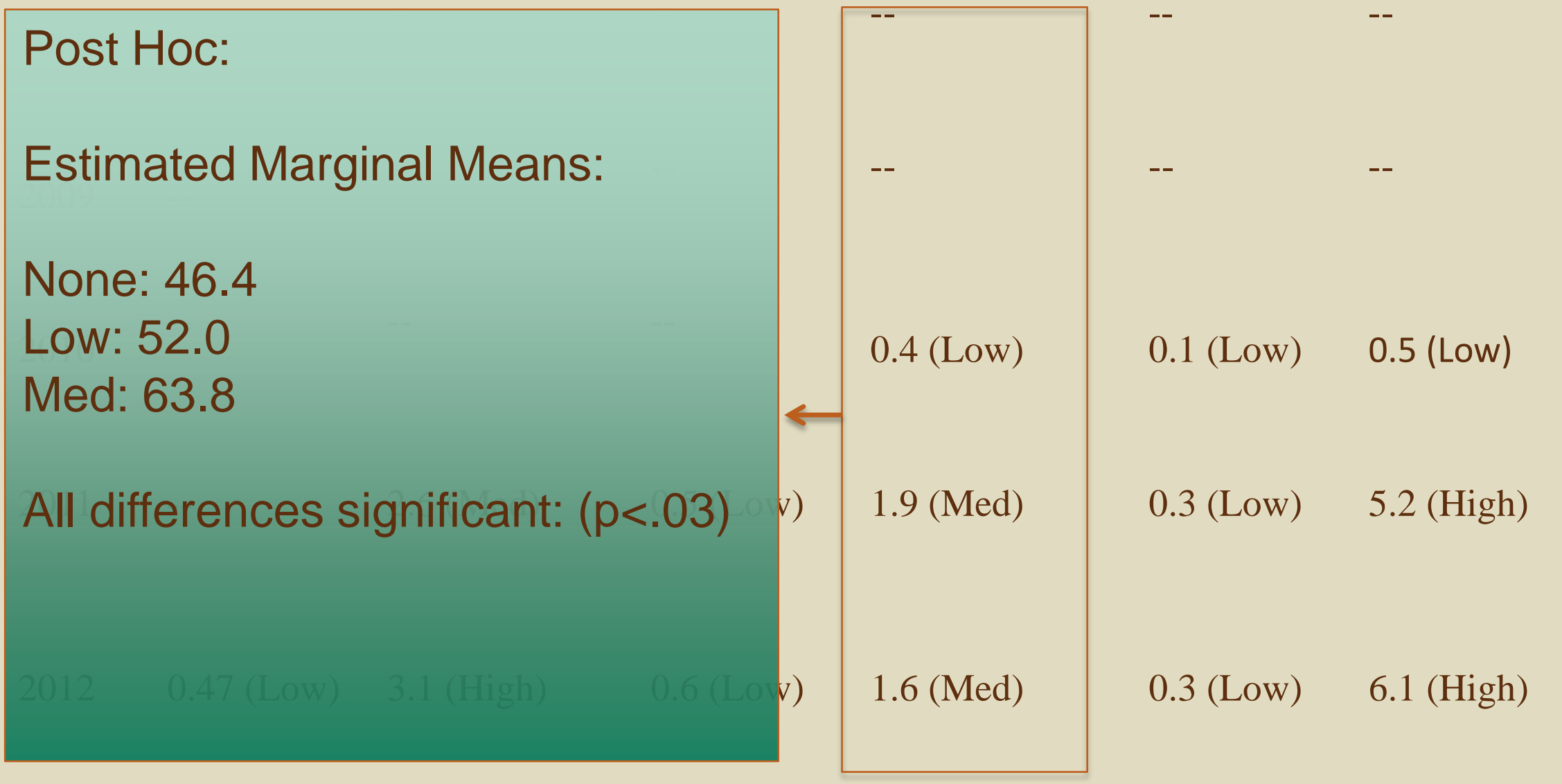

Note: -- Means that no lectures were viewed, either because they were not available or the instructor did not allow viewing. 


\section{Anatomy}

Class Anatomy Microbiology Pathology Pharmacology Physiology Overall

\begin{tabular}{|c|c|c|c|c|c|}
\hline & & -- & Differences were significant by & -- & -- \\
\hline 2008 & -- & & $\begin{array}{l}\text { lecture views level }(F=62.240 ; \\
p<.0005) \text {; }\end{array}$ & & \\
\hline 2009 & -- & -- & & -- & -- \\
\hline & & 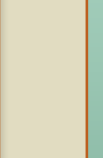 & $\begin{array}{l}\text { None: } 53.7(9.5) \\
\text { Low: } 60.7(9.0)\end{array}$ & & \\
\hline 2010 & -- & -- & & 0.1 (Low) & 0.5 (Low) \\
\hline 2011 & -- & $\begin{array}{l}\longrightarrow \\
2.6\end{array}$ & $\begin{array}{l}\text { Covariate (class) significant (F } \\
=11.48 ; p=.001)\end{array}$ & 0.3 (Low) & 5.2 (High) \\
\hline & & & Estimated Marginal Means: & & \\
\hline 2012 & 0.47 (Low) & 3.1 & $\begin{array}{l}\text { None: } 53.0(.476) \\
\text { Low: } 63.0(1.043)\end{array}$ & 0.3 (Low) & 6.1 (High) \\
\hline
\end{tabular}

Note: -- Means that no lectures were viewed, elther because they were not available or the instructor did not allow viewing. 


\section{Mixed Disciplines/Mixed Methods/Mixed Results}




\section{Microbiology}

Class Anatomy Microbiology Pathology Pharmacology Physiology Overall

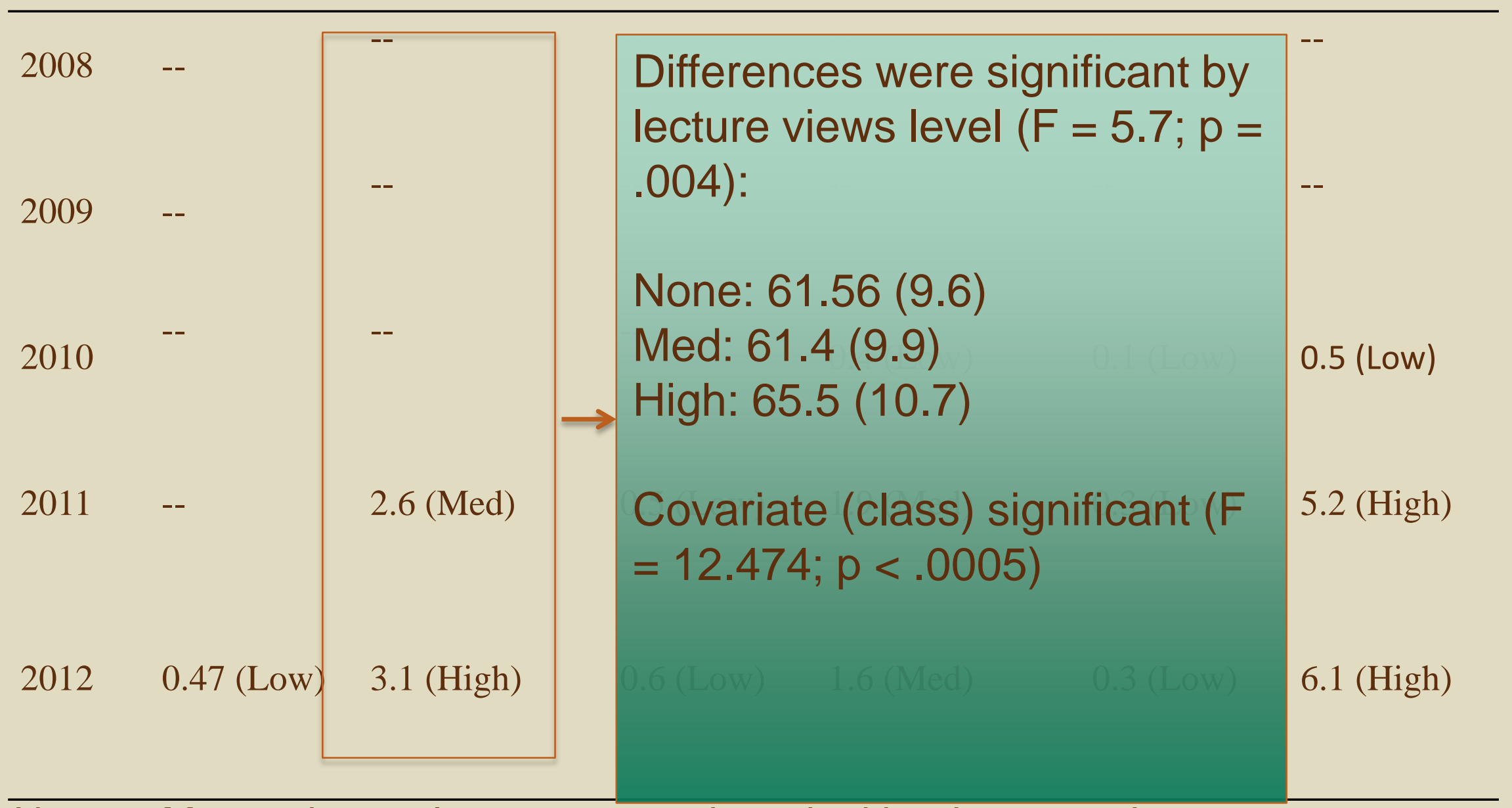

Note: -- Means that no lectures were viewed, either because they were not available or the instructor did not allow viewing. 


\section{Microbiology}

Class Anatomy Microbiology Pathology Pharmacology Physiology Overall

\begin{tabular}{|c|c|c|c|c|}
\hline 2008 & -- & & Post Hoc Analysis & \\
\hline 2009 & -- & -- & Estimated Marginal Means: & -- \\
\hline 2010 & -- & -- & $\begin{array}{l}\text { None: } 64.3 \\
\text { Med: } 59.4 \\
\text { High: } 61.1\end{array}$ & 0.5 (Low) \\
\hline 2011 & -- & 2.6 (Med) & $\begin{array}{l}\text { "None" significantly higher than } \\
\text { "Med"; No other differences } \\
\text { significant }\end{array}$ & 5.2 (High) \\
\hline 2012 & 0.47 (Low) & 3.1 (High) & .6 (Low) & 6.1 (High) \\
\hline
\end{tabular}

Note: -- Means that no lectures were viewed, either because they were not available or the instructor did not allow viewing. 


\section{Discussion}

- In two areas, use of lecture capture was not associated with any change in learning outcomes.

- Both areas basic science applied

- More mixed teaching methods other than straight lecture

- Less student use of lecture capture. 


\section{Discussion}

- In two areas, use of lecture capture associated with higher learning outcomes.

- Both areas rely heavily on lecture (as opposed to mixed lecture or group work.)

- Both areas are Basic Science - research orientation focus.

- Associated with high use (Pharmacology) or a student-led increase in use (Anatomy) which had not been permitted by instructors. 


\section{Discussion}

- One area (Microbiology) lecture capture use associated with higher means, but lower estimated marginal means when controlling for the covariate. The covariate had a more powerful effect on learning outcome than the lecture capture use, suggesting that some other factor contributed more to changes than did lecture capture. 


\section{Discussion}

- No evidence to suggest that incorporating lecture capture results in reduced learning.

- More research warranted to explore the possibility that use of captured lectures is beneficial, particularly with:

- Fact-focused "building-block" disciplines

- Classes relying primarily on lecture

- Students, in our context, good judges of when lecture capture will be helpful (?) 


\title{
Contact Information:
}

\author{
Jared Danielson \\ E-mail: jadaniel@iastate.edu
}

